\title{
tRNA fragments: novel players in intergenerational inheritance
}

Cell Research (2016) 26:395-396. doi:10.1038/cr.2016.24; published online 23 February 2016

Non-genetic inheritance is an evocative topic; in the past few years, the debate around potential inheritance of life-time experiences independent of social factors in mammals has become highly prominent due to increasing evidence for phenotypes in the offspring after paternal environmental exposures. Strikingly, two independent studies published in Science newly implicate a special class of RNA, transfer RNA fragments, in the intergenerational effects of paternal dietary intervention.

Histone post-translational modifications, DNA methylation and RNA underlie phenomena of non-genetic inheritance in plants and invertebrates [1]. The same potentially contribute to encoding and paternal transmission of information in mammals, which requires escaping of two big waves of epigenetic reprogramming that occur after fertilization and during sperm development, respectively [2]. Reprogramming enables return to pluripotency and is believed to enable a reset to a naïve state free of deleterious marks potentially caused by environmental impacts. Changes of epigenetic marks in germ cells have been reported after a range of environmental exposures, but conclusive proof for the direct involvement in information transfer across generations only exists for RNA. Injection of total sperm RNA of mice that had undergone early life stress into naïve zygotes was shown to recapitulate behavioral and metabolic alterations in the resulting offspring that were observed in progeny obtained by natural breeding [3]. The total sperm RNA displayed alterations in miRNA and a piRNA cluster.
A later study demonstrated that a similar set of miRNAs, found to be changed after chronic stress, was sufficient to induce changes in offspring when injected into zygotes [4]. Recently, Grandjean et al. [5] provided insight into the potential of high-fat/high-sugar diet-altered testis and/or sperm RNA and/or single miRNA to recapitulate a metabolic phenotype in the offspring obtained from RNAinjected naïve embryos.

Previously Sharma et al. [6] used a low-protein diet-based mouse model and observed a metabolic phenotype, a decreased cholesterol ester level, in the offspring of paternally exposed males. In the new paper published in Science, they used hepatic Squalene epoxidase (Sqle) expression in offspring resulting from IVF as a read-out for successful transmission of a phenotype. Consistent with a previous study [5], they report altered miRNA levels in sperm of males subjected to altered diet, but subsequently focused on tRNA fragments [7] which they found to be the most abundant class of RNA in mature sperm and therefore in their opinion have the highest chance to be functionally relevant in the zygote after fertilization.

tRNA recently gained attention in contexts other than protein translation. In 2013 it was first appreciated that tRNA fragments can exert regulatory functions on a mRNA target in a mammalian cell line after viral infection [8]. A recent study by Peng et al. [9] reported the presence of tRNA fragments in mature sperm. Sharma et al. [7] demonstrate that tRNA fragments (termed tRFs in the study) are delivered along with miRNAs to maturing spermatids during their transit through the epididymis, part of the male reproductive tract where sperm undergo maturation, in mice based on comparative RNA sequencing analysis of sperm isolated from caput and cauda epididymis. They propose epididymosomes as vehicles, exosome-like structures that protect RNA from degradation. To test this, they carried out epididymosome fusion assays. They incubated caput sperm with isolated epididymosomes and performed comparative sequencing of the sperm before and after the incubation. Their observations are consistent with previous bovine studies that describe the convey of small RNA by epididymosomes to developing sperm cells [10].

To investigate the functional relevance of tRFs, Sharma et al. [7] manipulated ES cells with LNA-based antisense oligonucleotides against tRF GlyGCC. They observed upregulation of several genes, which are targets of the endogenous retroelement MERVL. Interestingly, MERVL targets are highly expressed and derepressed after inhibition of tRF GlyGCC in early embryos, but downregulated in offspring of low protein-fed fathers. Inhibition of any other tRF-Gly isoacceptors in ES cells did not impact mRNA levels. Noteworthy, although tRF GlyGCC level increased in mature sperm after low-protein diet, such change was not statistically significant. Further studies might be required to determine whether their observations in ES cells and early embryos following GlyGCC inhibition are relevant to their animal model. When they injected either the sperm small RNA fraction of low protein-fed fathers or synthetic tRF-GlyGCC oligos to naïve zygotes, they observed similar 
alterations in expression changes of MERVL targets in early embryos to those in IVF offspring of low protein-fed fathers. To establish a connection between tRF-mediated regulation of gene expression in the embryo and the specifically gained tRFs during sperm transit through the epididymis, they report decreased MERVL target expression in zygotes generated by IVF with cauda sperm as compared to testicular sperm. Sharma et al. [7] interpret that solely sperm tRFs gained during spermatogenesis enable full elicitation of expression changes in offspring, while the study from Grandjean et al. [5] shows the induction of a full metabolic phenotype after total testis RNA injection.

Obvious next steps should involve the investigation of the biological consequences of altered MERVL target expression. An important side observation in the study is that the epigenetic effects of diet are outweighed by the effect of parent of origin. This might indicate that low-protein diet has no epidemiologically significant effect on the offspring.

Chen et al. [11] used a high-fat diet (HFD) and observed glucose intolerance and insulin resistance in the exposed animals and their paternal line offspring resulting from sperm head injection into normal mouse oocytes, indicating epigenetic transmission of metabolic disease. Consistent with the study by Sharma et al., they observed small RNA alterations in F0 sperm after dietary intervention and focused on changes in tRNA fragments that they term tsRNAs (tRNA-derived small RNAs). In addition, they detected an upregulation of $\mathrm{m} 5 \mathrm{C}$ and $\mathrm{m} 2 \mathrm{G}, 2$ methyl RNA modifications, in HFD sperm tsRNAs by LC-MS/MS. They demonstrate that the tsRNA-containing fraction but not the miRNA- or long RNA-containing fraction of sperm RNA is capable of transmitting glucose intolerance to the offspring using a series of RNA injection experiments into naive zygotes. tsRNA modifications seem to be crucial for the information transfer since they did not observe such effects using synthetic tsRNAs. This is consistent

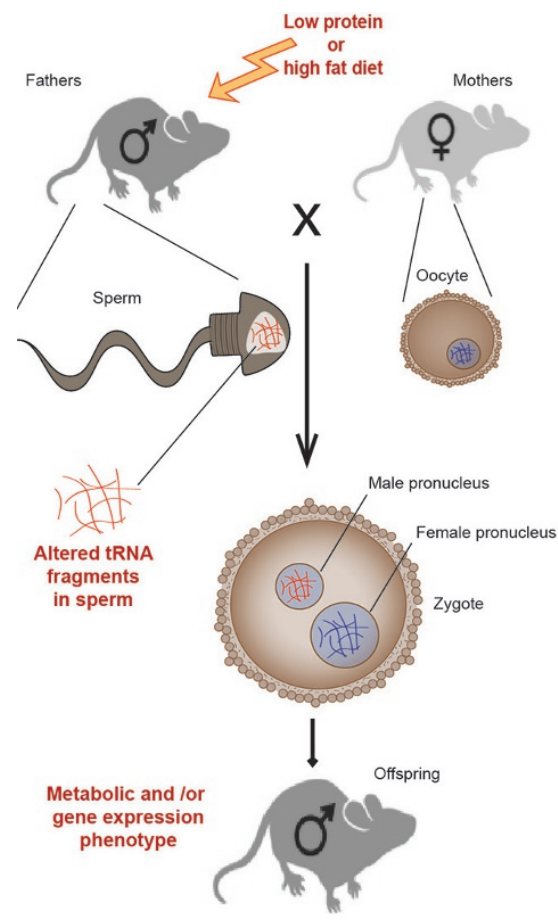

Figure 1 tRNA-derived fragments mediate intergenerational inheritance of diet-induced metabolic alterations.

with a previous study by Kiani et al. [12] showing that tRNA modifications are necessary for RNA-mediated epigenetic heredity of a genetically induced trait. Chen et al. speculate that increased methyl modifications might affect tsRNA stability.

Paternal high-fat diet affected pancreatic DNA methylation and gene expression in the offspring, but the changes did not correlate. The observed DNA methylation changes might account for the transmission of insulin resistance, an effect absent in offspring generated by tsRNA or total RNA injection. RNA sequencing of 8-cell embryos resulting from injection of sperm tsRNA fractions from HFD-fed fathers revealed dysregulated expression of a host of genes, some of which with metabolic function. Since their promoter sequences matched with differentially expressed tRNA fragments, the authors speculate a transcriptional cascade effect initiated by paternal tsRNA, ultimately causing metabolic changes in adulthood.

Overall, both studies provide evidence for tRNA fragments as players in epigenetic transmission of information. They conclude that diet-induced changes in tRNA fragments account for nutritioninduced transgenerational effects (Figure 1). This conclusion might be of particular relevance to human pathophysiology, since a recent study observed alterations in germline non-coding RNA of obese men [13]. Interestingly, data from zygotic injection experiments of synthetic tRNA fragments lacking post transcriptional modifications suggest that these modifications are indispensible to induce transgeneratioanal effects [11] while Sharma's study [7] suggests functionality of unmodified tRNA fragments. It remains to be seen how tsRNAs might act in embryos to relay information to the next generation.

\section{Katharina Gapp ${ }^{1,2}$, Eric A Miska ${ }^{1,2}$ \\ ${ }^{1}$ The Gurdon Institute and Department of Genet- ics, University of Cambridge, Tennis Court Road, Cambridge, CB2 1QN, UK; ${ }^{2}$ The Wellcome Trust Sanger Institute, Wellcome Trust Genome Cam- pus, Hinxton, Cambridge, CB10 1SA, UK \\ Correspondence: Eric A Miska \\ E-mail: eam29@cam.ac.uk}

\section{References}

1 Heard E, Martienssen RA. Cell 2014; 157:95109.

2 Bohacek J, Mansuy IM. Nat Rev Genet 2015; 16:641-652.

3 Gapp K, Jawaid A, Sarkies P, et al. Nat Neurosci 2014; 17:667-669.

4 Rodgers AB, Morgan CP, Leu NA, et al. Proc Natl Acad Sci USA 2015; 112:13699-13704.

5 Grandjean V, Fourré S, De Abreu DA, et al. Sci Rep 2015; 5:18193.

6 Carone BR, Fauquier L, Habib N, et al. Cell 2010; 143:1084-1096.

7 Sharma U, Conine CC, Shea JM, et al. Science 2015; 351:391-396.

8 Wang Q, Lee I, Ren J, et al. Mol Ther 2013; 21:368-379.

9 Peng H, Shi J, Zhang Y, et al. Cell Res 2012; 22:1609-1612.

10 Belleannée C, Calvo É, Caballero J, et al. Biol Reprod 2013; 89:30.

11 Chen Q, Yan M, Cao Z, et al. Science 2015; 351:397-400.

12 Kiani J, Grandjean V, Liebers R, et al. PLoS Genet 2013; 9:e1003498.

13 Donkin I, Versteyhe S, Ingerslev LR, et al. Cell Metab 2015; 23:369-378.

Cell Research | Vol 26 No 4 | April 2016 